\title{
Synthesis of ZnO submicron spheres by a two-stage solution method
}

\author{
Xinghua Zhan · Fei Chen $\cdot$ Zoran Salcic $\cdot$ \\ Chee Cheong Wong $\cdot$ Wei Gao
}

Received: 29 September 2011 / Accepted: 12 October 2011/Published online: 5 November 2011

(C) The Author(s) 2011. This article is published with open access at Springerlink.com

\begin{abstract}
This paper presents a two-stage method to synthesize the mono-dispersed $\mathrm{ZnO}$ spheres. The size and uniformity of $\mathrm{ZnO}$ spheres can be controlled in the submicrometer range. This makes the method applicable to the fabrication of photonic crystal structures. The effects of different reaction parameters such as reaction temperatures, reaction times, and water concentrations on the formation of $\mathrm{ZnO}$ spheres were investigated. On the basis of the experimental results, we discuss and propose a possible mechanism to elucidate the formation of $\mathrm{ZnO}$ submicron spheres. Furthermore, the room temperature photoluminescence (PL) measurements of the products were carried out to investigate their optical properties. The PL results showed that the $\mathrm{ZnO}$ submicron spheres exhibited a strong ultraviolet emission and a broadband green emission in the visible region.
\end{abstract}

Keywords $\mathrm{ZnO}$ spheres - Photonic crystal structure . Reaction parameters · Photoluminescence

\section{Introduction}

Zinc oxide $(\mathrm{ZnO})$ is an important wide band gap semiconductor material due to its numerous attractive properties such

X. Zhan · F. Chen · Z. Salcic $(\bowtie) \cdot$ W. Gao $(\square)$

The University of Auckland, PB 92019, Auckland 1142,

New Zealand

e-mail: z.salcic@auckland.ac.nz

W. Gao

e-mail: w.gao@auckland.ac.nz

C. C. Wong

Nanyang Technological University Singapore, Singapore, Singapore as high excitation binding energy, controllable conductivity, and transparency in the visible light (Wang 2004). As such, it has attracted much interest because of the potentially wide applications in electronics and optoelectronics.

Diverse methods have been developed in the synthesis of different $\mathrm{ZnO}$ nano- or micro-structures. However, largescale applications depend on the development of simple and low-cost production procedures. High-temperature vapour deposition and solution-based methods have been employed for preparing $\mathrm{ZnO}$ materials of various sizes and morphologies. The vapour deposition route is generally used for the fabrication of $\mathrm{ZnO}$ nanostructures. It requires a relatively high temperature, expensive vacuum equipment, and complex procedures, which restrict the use in practical applications (Ramgir et al. 2006; Khomenkova et al. 2007). Compared with the high-temperature vapour deposition method, solution-based methods are more effective in synthesizing $\mathrm{ZnO}$ material with controllable morphology. This method also allows for the growth of $\mathrm{ZnO}$ nano- or microstructures at a relatively low temperature. Furthermore, the solution-based methods are relatively economic and can be applied to large-scale productions (Peng et al. 2006; Yu et al. 2005).

Materials with low crystal symmetry have been used as building blocks in bottom-up self-assembly. For this reason, the spherical-shaped structure is the most favourable morphology due to its high level of symmetry. However, wurtzite $\mathrm{ZnO}$ prefers to grow along the $c$-axis in solution media because of its noncentrosymmetric hexagonal structure, which results in the commonly observed onedimensional (1-D) $\mathrm{ZnO}$ nanostructures such as nanorods and nanowires (Yan et al. 2008; Gao and Li 2009). To overcome this problem, researchers used surfactants as crystal growth modifiers to obtain $\mathrm{ZnO}$ spheres. For example, it was reported that polyethylene glycol (PEG)

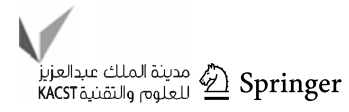


and polyvinylpyrrolidone (PVP) could be used in a solution-based process as surfactants (Wang et al. 2007; Deng et al. 2008). Nano-composite spheres of $\mathrm{ZnO} / \mathrm{PVP}$ were first synthesized and the PVP phase was removed by subsequent heating. However, this method is complicated and expensive.

In this paper, we present a simple and low-cost method to synthesize mono-dispersed $\mathrm{ZnO}$ spheres with good uniformity, which are suitable for the fabrication of photonic crystal structures. We also investigated the effects of processing parameters that can affect the morphology of the $\mathrm{ZnO}$ particles. Furthermore, based on the experimental results, we propose a possible mechanism to elucidate the formation of $\mathrm{ZnO}$ spheres.

\section{Experimental design}

Spray pyrolysis and atomic layer epitaxy methods have been employed to synthesize spherical $\mathrm{ZnO}$ particles by allowing zinc acetate $\left[\mathrm{Zn}(\mathrm{Ac})_{2}\right]$ to react with water vapour (Alver et al. 2007; Kowalik et al. 2009). From this point, if $\mathrm{Zn}(\mathrm{Ac})_{2}$ and water are dissolved in a solvent with a sufficiently high boiling point, hydrolyzation will occur and the precipitation of $\mathrm{ZnO}$ will be obtained. Furthermore, the particles are generally formed by nucleation, growth, and aggregation in the solvent. These three processes, which decide the particle morphologies and size, can be controlled by the reaction conditions.

Polyols are considered good candidates for solvents as they can easily dissolve various organic and inorganic compounds, and they have relatively high boiling points. Moreover, they have fairly high dielectric constants. These properties make the reactions possible at the temperatures above $100^{\circ} \mathrm{C}$.

We used diethylene glycol (DEG) as the solvent and the surfactant, and further investigated the effects of other parameters in the reactions that can affect the morphologies of the $\mathrm{ZnO}$ particles such as reaction temperatures, reaction times, water concentrations, etc.

\section{Experiment procedures}

A two-stage solution-based method has been used to synthesize the $\mathrm{ZnO}$ spheres, which provides an effective and easy way to prepare spherical $\mathrm{ZnO}$ particles with good uniformity. The experimental procedure is described as follows.

In the first stage, $0.003 \mathrm{~mol} \mathrm{Zn}(\mathrm{Ac})_{2} \cdot 2 \mathrm{H}_{2} \mathrm{O} /$ zinc nitrate $\left[\mathrm{Zn}\left(\mathrm{NO}_{3}\right)_{2} \cdot 6 \mathrm{H}_{2} \mathrm{O}\right]$ was added to $30 \mathrm{ml}$ DEG in a $50 \mathrm{ml}$ beaker. The solution was then heated on a hot plate at the temperature of $50^{\circ} \mathrm{C}$ with magnetic stirring until a transparent solution was achieved. The solution was transferred to $125 \mathrm{ml}$ autoclave from the beaker. Then, the sealed autoclave was heated in an electrical oven under a certain temperature for several hours. After that, the autoclave was cooled down to room temperature in air. The solid phase formed was then separated by centrifugation and washed with ethanol. The supernatant, including materials such as DEG, dissolved reaction products and un-reacted source materials, was decanted off and saved for use in the second stage.

In the second stage, $0.003 \mathrm{~mol} \mathrm{Zn}(\mathrm{Ac})_{2} \cdot 2 \mathrm{H}_{2} \mathrm{O} /$ $\mathrm{Zn}\left(\mathrm{NO}_{3}\right)_{2} \cdot 6 \mathrm{H}_{2} \mathrm{O}$ was added to $30 \mathrm{ml} \mathrm{DEG}$ in a $50 \mathrm{ml}$ beaker. The solution was then heated on the hot plate at $50^{\circ} \mathrm{C}$, and some volume $(1-10 \mathrm{~mL})$ of the primary supernatant obtained from the first stage was added to it. The solution was then stirred by a magnetic stirrer until it became clear. The remaining steps are the same as described in the first stage.

Silicon ( $\mathrm{Si}$ ) substrates were firstly cleaned in acetone with ultrasonic bath for $10 \mathrm{~min}$, and then rinsed in de-ionized (DI) water and heated at $200^{\circ} \mathrm{C}$ for an hour to remove contaminants. For preparing samples, one drop of the resultant $\mathrm{ZnO}$ colloidal spheres were dipped onto $\mathrm{Si}$ substrates to form a film which was subsequently heated at $200^{\circ} \mathrm{C}$ for an hour. Finally, the samples were dried at $400^{\circ} \mathrm{C}$ for $2 \mathrm{~h}$ in air.

\section{Experimental results}

Particle size and morphology were characterized by field emission scanning electron microscopy (FESEM). X-ray diffraction (XRD) was used to analyze the crystal structure and identify the phases. PL measurements were carried out by using FLUOROLOG-3-TAU at room temperature. As discussed in "Experimental design", the $\mathrm{ZnO}$ precipitation in our experiments is formed by hydrolyzation. The DEG is a non-ionic solvent agent; and the hydroxyl group (-OH) in the reactions is provided by the hydrous compounds within the source materials. The volume ratio of water content will affect the morphology of the products. As the hydrolyzation occurs in a sufficiently high boiling point, the reaction temperature and time will be crucial factors in our experiments. The effects of various processing parameters on the morphology and size of $\mathrm{ZnO}$ are presented below.

\section{The effect of hydrate}

Hydrous compound in the source material $\mathrm{Zn}(\mathrm{Ac})_{2}$ or $\mathrm{Zn}\left(\mathrm{NO}_{3}\right)_{2}$ is denoted by the term $\cdot n \mathrm{H}_{2} \mathrm{O}$, where $n$ is the number of water molecules per molecule of salt. In order to investigate the effects of hydrous compound in the source material, the experiment was started from a solution of dissolving dehydrated $\mathrm{Zn}(\mathrm{Ac})_{2} / \mathrm{Zn}\left(\mathrm{NO}_{3}\right)_{2}$ into the solvent of dehydrated DEG. In this experiment, we found that $\mathrm{ZnO}$ 
was not obtained. As a result, the mechanism of hydrolysis for the $\mathrm{ZnO}$ formation in our experiments is confirmed.

To study the influence of the amount of water molecules, denoted as $n$, the $n$ was varied in the range of $0-6$. It was found that the precipitation of $\mathrm{ZnO}$ with nice spherical shape was obtained when $n=2$, which corresponds to the commercial salt $\mathrm{Zn}(\mathrm{Ac})_{2} \cdot 2 \mathrm{H}_{2} \mathrm{O}$. For the other cases $0 \leq n<2$ or $2<n \leq 6$, the shapes of $\mathrm{ZnO}$ particles became irregular. Figure 1 illustrates the FESEM images of $\mathrm{ZnO}$ spheres, which were obtained under the same reaction conditions for the case of $n=2$ and $n=6$.

The effect of solvent

For studying the effects of solvents in our research, DEG, pure or mixed with other materials, was used in our experiments. Ethylene Glycol (EG) was also used as the solvent to compare with DEG. By dissolving $\mathrm{Zn}(\mathrm{Ac})_{2} \cdot 2 \mathrm{H}_{2} \mathrm{O}$ /
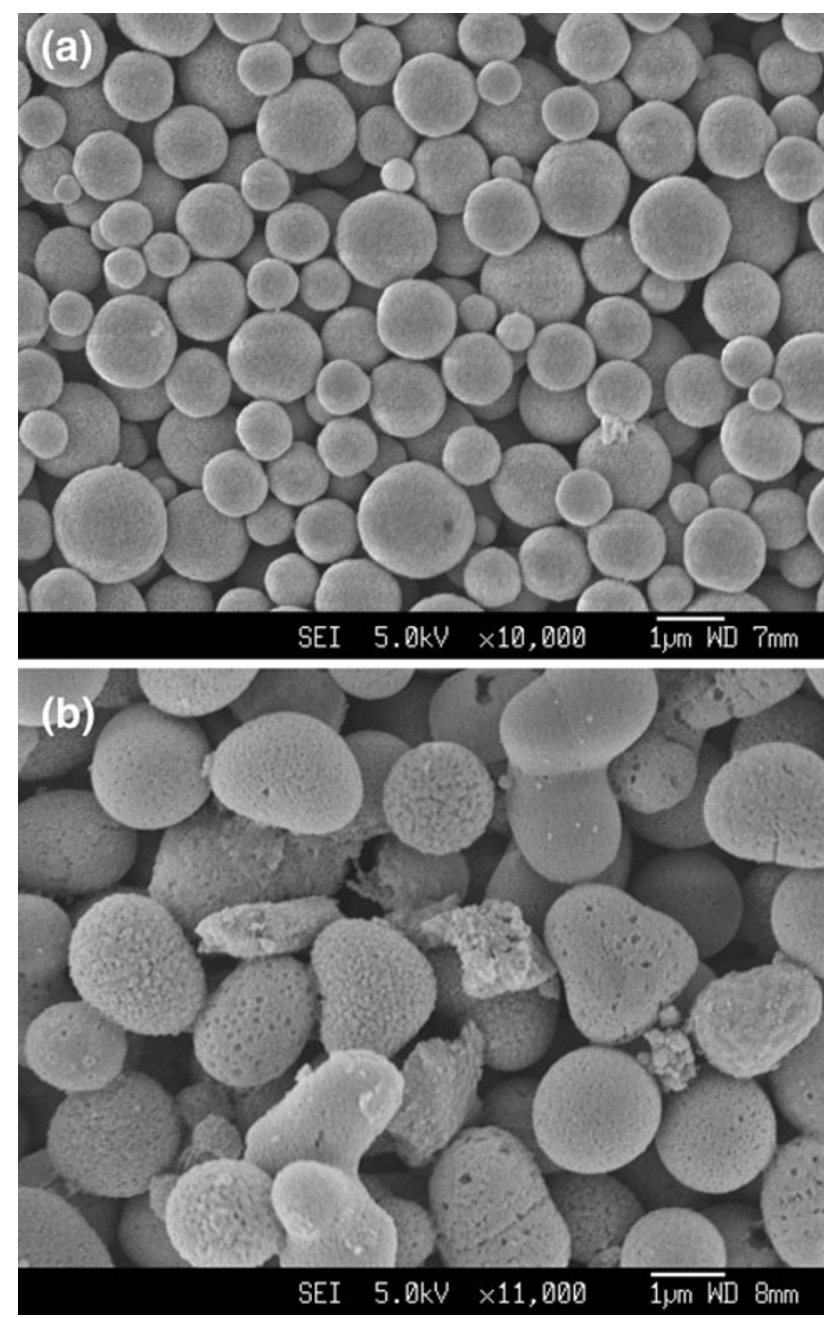

Fig. 1 FESEM images of a $\mathrm{ZnO}$ spheres obtained when $n=2$, and b $\mathrm{ZnO}$ spheres obtained when $n=6 . n$ is the water molecular number in $\mathrm{Zn}(\mathrm{Ac})_{2} \cdot n \mathrm{H}_{2} \mathrm{O}$
$\mathrm{Zn}\left(\mathrm{NO}_{3}\right)_{2} \cdot 6 \mathrm{H}_{2} \mathrm{O}$ into EG and heating up the solution in the autoclave, $\mathrm{ZnO}$ precipitation was obtained, but with irregular morphologies. DEG was further mixed with EG to obtain different solvent characteristics in our experiments. Although this led to the formation of $\mathrm{ZnO}$, the particles were dispersed with irregular shapes.

The precipitation of $\mathrm{ZnO}$ also took place when acetic acid was added into DEG instead of EG. It is proposed that water was produced by esterification between polyol and acetic acid during the reactions. However, it was found that the $\mathrm{ZnO}$ particles had irregular shapes. In the case of DEG mixed with a small amount of water (1-10\%) to form the solvents, the $\mathrm{ZnO}$ formed and had a rod-shape. Figure 2 shows the FESEM images of $\mathrm{ZnO}$ particles, which were obtained by using different solvents.

A small amount of primary supernatant, which was obtained and saved in the first stage, was added into DEG to form the solvents. The mono-dispersed $\mathrm{ZnO}$ spheres were formed with diameter of submicron size and good uniformity. XRD analysis of the $\mathrm{ZnO}$ colloid revealed that the material has hexagonal $\mathrm{ZnO}$ phase with no preferential growth directions. This result provides a method to synthesize mono-dispersed $\mathrm{ZnO}$ colloidal spheres over a broad size range (500-1,000 $\mathrm{nm}$ ) with good control over their diameter. It has been reported that the particles with the size in the range of submicron can be used to create photonic crystals with the band gap covering the entire visible spectrum, and also extending well into the ultraviolet (UV) and infrared (IR) ranges (Seelig et al. 2003).

\section{The effect of salt concentration}

When the salt concentration is lower than $0.1 \mathrm{~mol} / \mathrm{l}$, the $\mathrm{ZnO}$ spherical mono-dispersed particles with one micrometer size were obtained. In the range of $0.1-0.2 \mathrm{~mol} / 1$, the $\mathrm{ZnO}$ particles became irregular and larger. Figure 3 shows a FESEM image of $1 \mu \mathrm{m}$ sized $\mathrm{ZnO}$ spheres obtained with a salt concentration of $0.1 \mathrm{~mol} / \mathrm{l}$.

The effects of reaction temperature and time

In order to analyze the effect of reaction temperature on the formation of $\mathrm{ZnO}$ spheres, we varied the reaction temperature from 160 to $250^{\circ} \mathrm{C}$. In this temperature range, the morphology (spherical shape) of $\mathrm{ZnO}$ particle remains almost unchanged. When the reaction temperature is in the range from 100 to $150^{\circ} \mathrm{C}$, no $\mathrm{ZnO}$ spheres formed.

Under a fixed reaction temperature, the reaction time was varied from 6 to $24 \mathrm{~h}$. The results indicated that the shape and size of $\mathrm{ZnO}$ particles remain similar. However, in order to obtain better uniformity of the products, a reaction time longer than $6 \mathrm{~h}$ is required. $\mathrm{ZnO}$ spherical particles do not form with a short reaction time of 2 to $6 \mathrm{~h}$. 

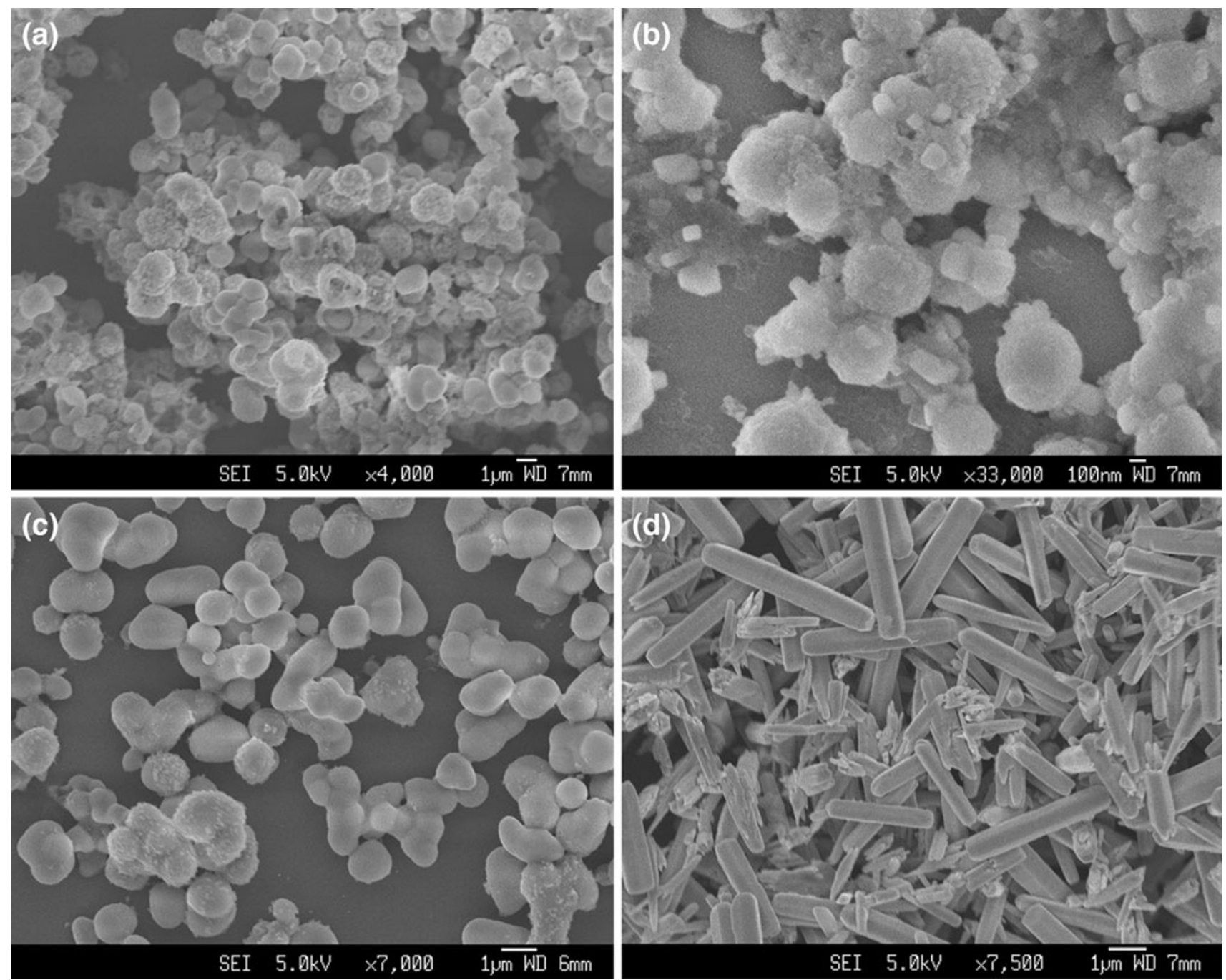

Fig. 2 FESEM images of a ZnO particles obtained by using EG as solvent, b ZnO particles obtained by using DEG mixed with EG, $\mathbf{c}$ ZnO particles obtained by using DEG mixed with acetic acid, and $\mathbf{d} \mathrm{ZnO}$ particles obtained by using DEG mixed with $5 \%$ water

Figure 4 shows the FESEM images of $\mathrm{ZnO}$ spheres obtained at the reaction temperature of $200^{\circ} \mathrm{C}$ with two different reaction times of 6 and $12 \mathrm{~h}$.

\section{Final product characteristics}

The characteristics of the particles obtained under the optimal conditions are described below. The products were synthesized using $\mathrm{Zn}(\mathrm{Ac})_{2} \cdot 2 \mathrm{H}_{2} \mathrm{O}$ as the source material and DEG as the solvent (salt concentration of $0.1 \mathrm{~mol} / \mathrm{l}$ ). The reaction temperature was $200^{\circ} \mathrm{C}$ and the reaction time was $12 \mathrm{~h}$. Phase composition and phase purity of the obtained products were identified by XRD (Fig. 5). Relatively strong and sharp peaks in the XRD pattern confirm that the products are $\mathrm{ZnO}$. The morphology of products was investigated by FESEM technique.

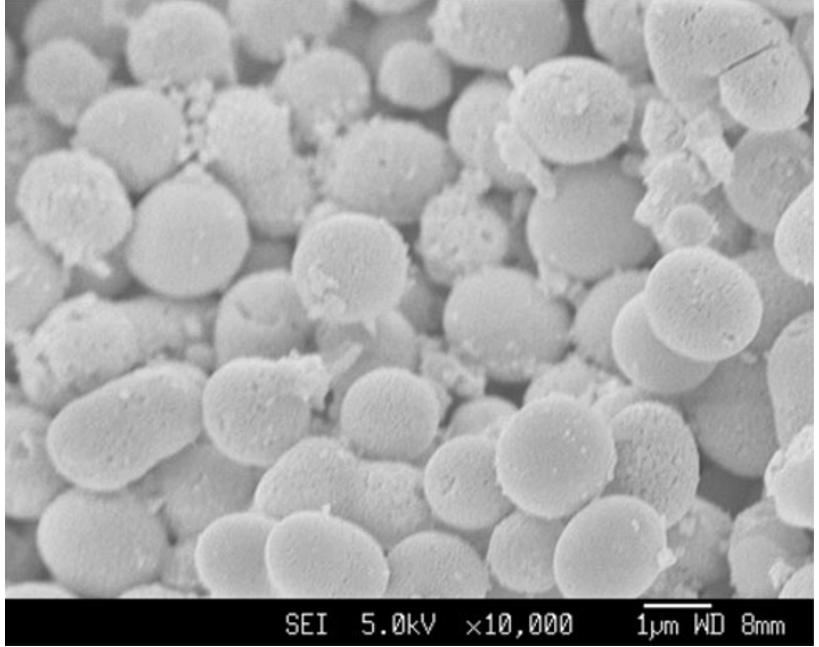

Fig. 3 A FESEM image of $1 \mu \mathrm{m}$ sized $\mathrm{ZnO}$ spheres obtained with $\mathrm{Zn}\left(\mathrm{NO}_{3}\right)_{2} \cdot 6 \mathrm{H}_{2} \mathrm{O}$ concentration of $0.1 \mathrm{~mol} / \mathrm{l}$ 

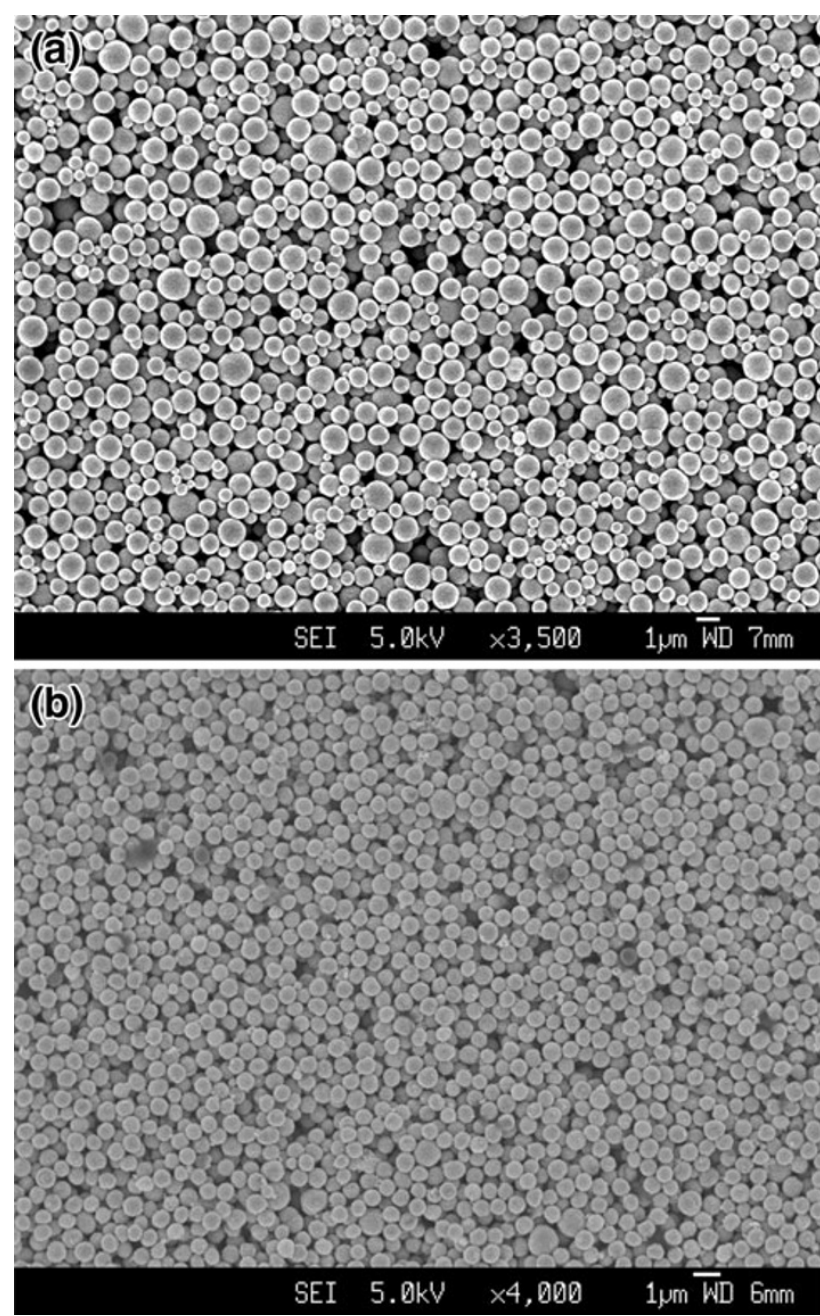

Fig. 4 FESEM images of a $\mathrm{ZnO}$ spheres obtained after reaction for $6 \mathrm{~h}$, and b $\mathrm{ZnO}$ spheres obtained after reaction of $12 \mathrm{~h}$

Figure 6a shows the $\mathrm{ZnO}$ particles are non-agglomerated and uniform with spherical shape. The sizes of these particles are mainly in a range of $480-500 \mathrm{~nm}$. In this micrograph, we can see that the $\mathrm{ZnO}$ particles were self- assembly to form a film with a good order. This makes it has the potential for construction of photonic crystal structure for controlling the wavelength of light emission.

From Fig. 6b, it can be seen that the $\mathrm{ZnO}$ spheres contain nano-structure, which indicates that the spheres are not single crystals but contain many smaller grains. In addition, energy dispersive spectroscopy (EDS) results of the products shown in Table 1 confirm that the spheres contain only $\mathrm{ZnO}$.

Figure 7 shows the room temperature photoluminescence (PL) emission spectrum of the hydrothermally synthesized $\mathrm{ZnO}$ particles excited by the laser with a wavelength of $325 \mathrm{~nm}$. A strong UV emission $(\sim 375 \mathrm{~nm}$ or $\sim 3.3 \mathrm{eV}$ ) and a broadband green emission centered at the wavelength of $565 \mathrm{~nm}$ (or $\sim 2.19 \mathrm{eV}$ ) in the visible region were observed from the PL results. The broadband green emission indicates that several defects may co-exist in the synthesized $\mathrm{ZnO}$ particles, including oxygen vacancy $\left(\mathrm{O}_{\mathrm{v}}\right)$, oxygen interstitials $\left(\mathrm{O}_{\mathrm{i}}\right)$, $\mathrm{Zn}$ vacancy $\left(\mathrm{Zn}_{\mathrm{v}}\right)$, and $\mathrm{Zn}$ interstitial $\left(\mathrm{Zn}_{\mathrm{i}}\right)$. According to the theoretical calculations of the intrinsic defects in $\mathrm{ZnO}$ using full-potential linear muffin-tin orbital method (FP-LMTO) (Peng et al. 2008), a figure is drafted to illustrate the defect energy levels in $\mathrm{ZnO}$ particles (Fig. 8). From this figure, we consider that the green emission was mainly attributed to $\mathrm{O}_{\mathrm{v}}$ in the synthesized $\mathrm{ZnO}$ particles as its energy level was very close to the defect energy level of $\mathrm{O}_{\mathrm{v}}(\sim 2.0 \mathrm{eV})$. The UV emission in the synthesized $\mathrm{ZnO}$ particles is very close to the $\mathrm{ZnO}$ bandgap $(\sim 3.37 \mathrm{eV})$; it can be attributed to the exciton radiate recombination ( $\mathrm{Wu}$ et al. 2008).

\section{Formation mechanism of the $\mathrm{ZnO}$ spheres}

To understand the growth mechanism of $\mathrm{ZnO}$ spheres, the growth process was analyzed based on the observations of the products at different reaction temperatures and times. A mechanism called oriented aggregation for the formation of
Fig. 5 XRD spectra of $\mathrm{ZnO}$ spheres obtained at $200^{\circ} \mathrm{C}$ for $12 \mathrm{~h}$, showing a polycrystalline structure

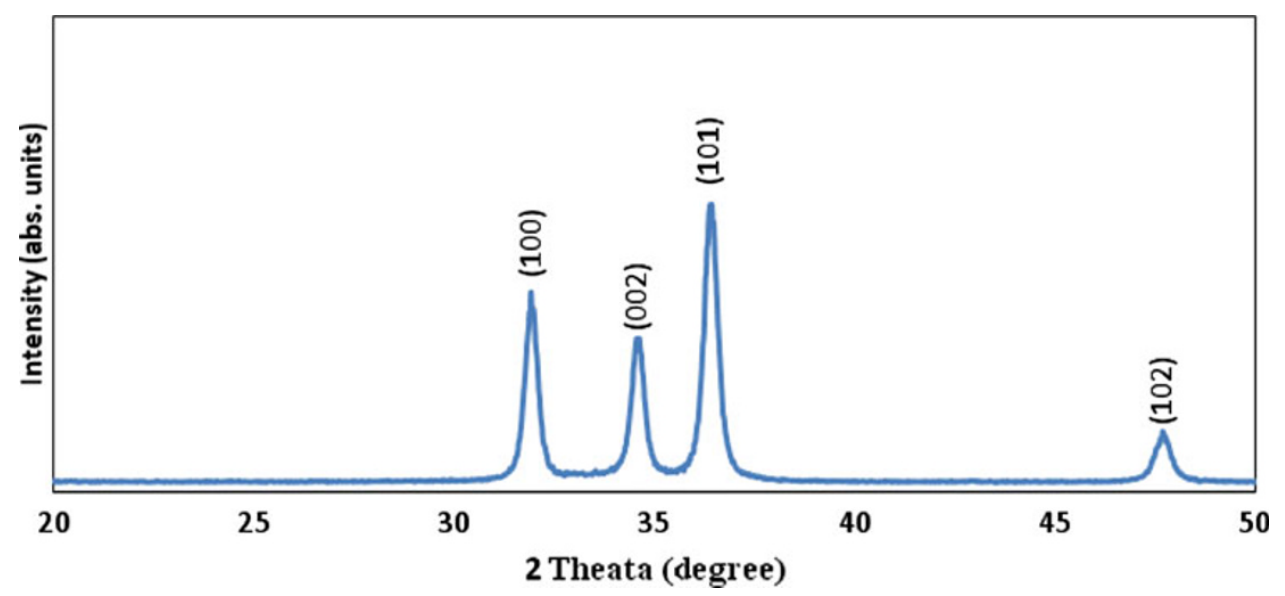

2 Theata (degree) 

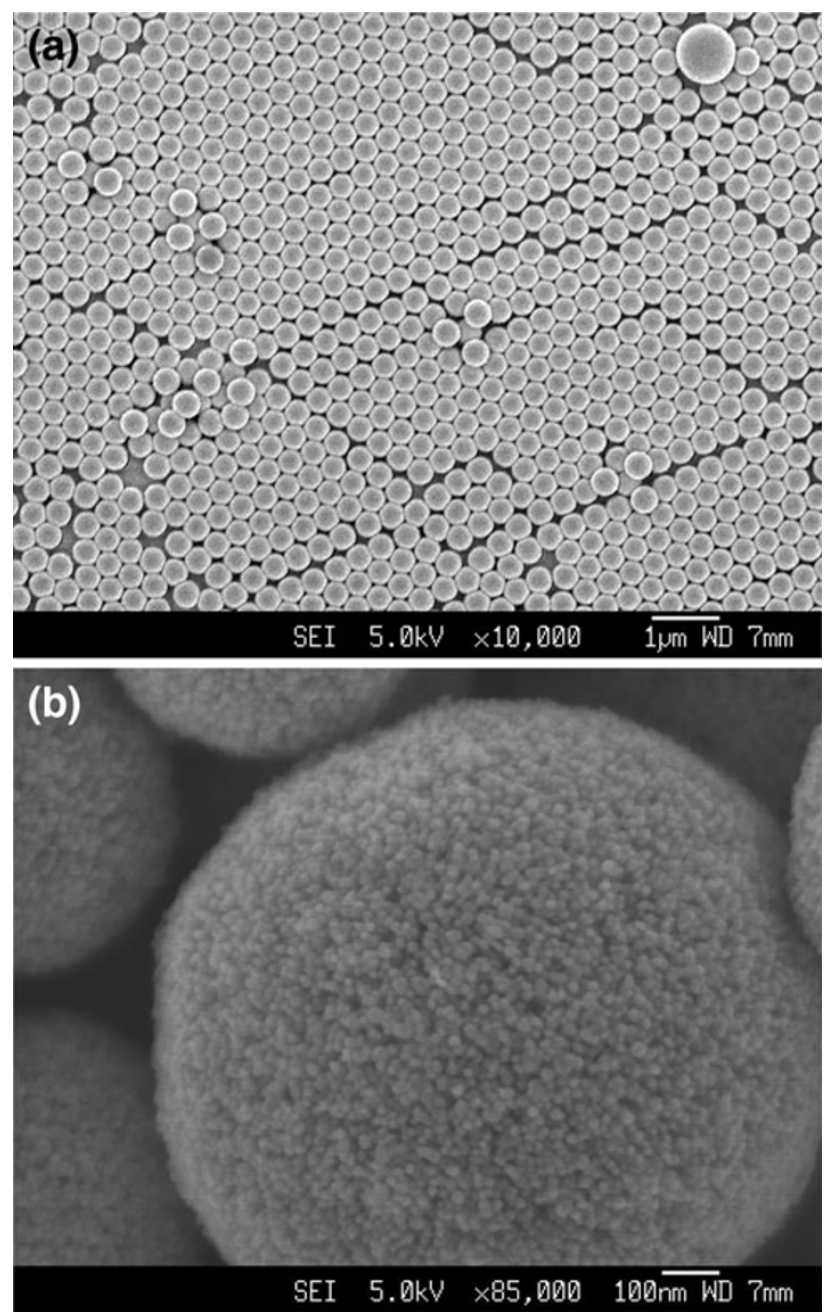

Fig. 6 FESEM images: a general view of the final product; b specified view of the final product

Table 1 EDS results of the products

\begin{tabular}{lcc}
\hline Element & Weight $(\%)$ & Atomic $(\%)$ \\
\hline $\mathrm{O}$ & 15.31 & 42.09 \\
$\mathrm{Si}$ & 1.01 & 1.58 \\
$\mathrm{Zn}$ & 83.68 & 56.33 \\
Totals & 100.00 & \\
\hline
\end{tabular}

complex structures (Ni et al. 2007) has been suggested. This mechanism proposes an oriented aggregation, which is regarded as one of the non-classical crystallization mechanisms. It leads to the formation of multiform crystal structures and morphologies. Similarly, self-assembled structures were reported in $\alpha-\mathrm{Fe}_{2} \mathrm{O}_{3}$ particles by Sugimoto et al. 1998 and Park et al. 1996. It is also reported that the sticking point in the synthesis of $\mathrm{Fe}_{3} \mathrm{O}_{4}$ sphere-like aggregates was the application of polyethylene glycol nonylphenyl ether and cyclodextrin (Xia et al. 2007). A

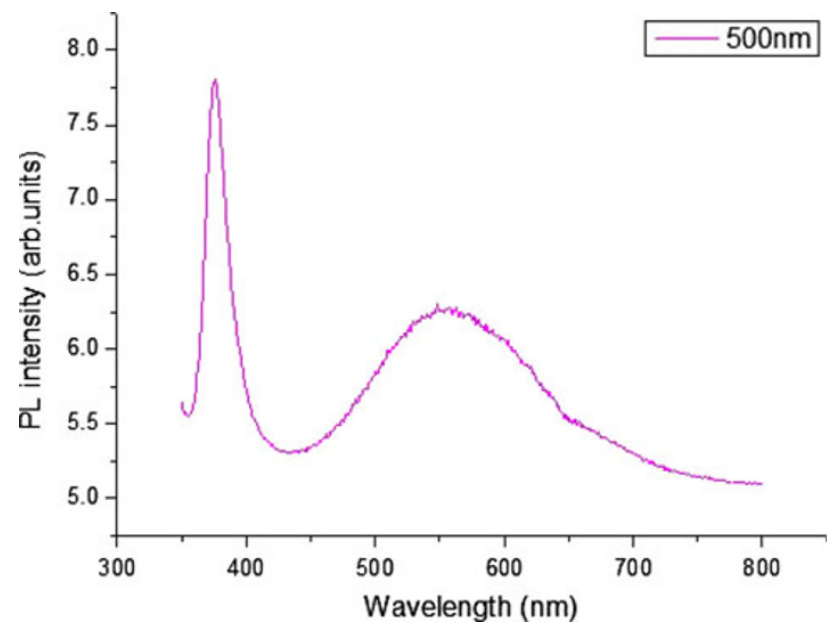

Fig. 7 Room temperature PL results of the $\mathrm{ZnO}$ spheres obtained at $200^{\circ} \mathrm{C}$ for $12 \mathrm{~h}$ excited with a laser of $325 \mathrm{~nm}$ wavelength

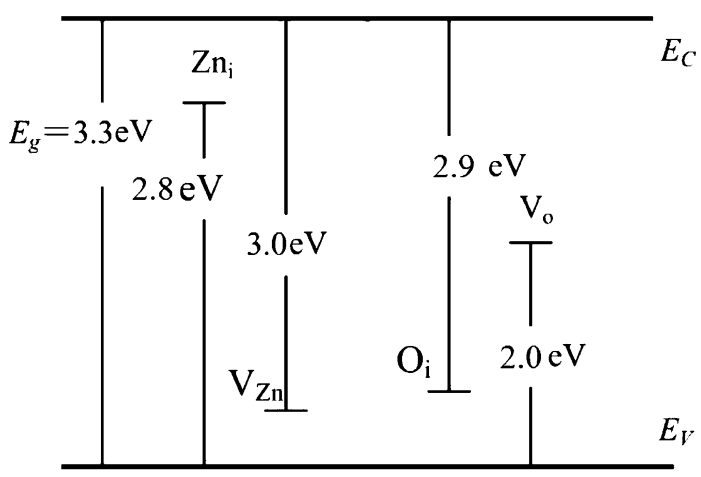

Fig. 8 The energy levels of various defects in $\mathrm{ZnO}$

similar formation mechanism that explains the $\mathrm{ZnO}$ sphere formation is therefore considered and proposed in our work.

DEG is a non-ionic surface active agent with formula of $\mathrm{O}\left(\mathrm{CH}_{2} \mathrm{CH}_{2} \mathrm{OH}\right)_{2}$. In the reaction, $\mathrm{Zn}^{2+}$ ions are attracted to the oxygen atoms on the DEG. The hydroxyl groups $(-\mathrm{OH})$ on the compound $\mathrm{Zn}(\mathrm{OH})_{2}$ can interact with each other, and as a result it provides a linkage or a bridge to the neighboring $\mathrm{Zn}(\mathrm{OH})_{2}$ as illustrated in Fig. 9. The clusters of $\mathrm{ZnO}$ nano-particles are then aggregated together to form a spherical shape to minimize the cluster surface energy. Figure 9 illustrates a schematic drawing of the formation process of the $\mathrm{ZnO}$ nano/micro-spheres. The corresponding equations are given below.

In our synthetic process, it is proposed that the complex compound $\left[\mathrm{Zn}(\mathrm{OH})_{2}\right]_{n}$ is formed by reaction 1 . Then, DEG coordinates with $\left[\mathrm{Zn}(\mathrm{OH})_{2}\right]_{n}$, and as a result the complex compound of $\left[\mathrm{Zn}(\mathrm{OH})_{2}-\mathrm{DEG}\right]_{n}$ is formed. These complex compounds are then broken up to release $\mathrm{H}_{2} \mathrm{O}$. The $\mathrm{ZnO}$ nano-particles then formed by reaction 3 . 
Fig. 9 Schematic illustration of the formation mechanism of the as-obtained $\mathrm{ZnO}$ nano/microspheres
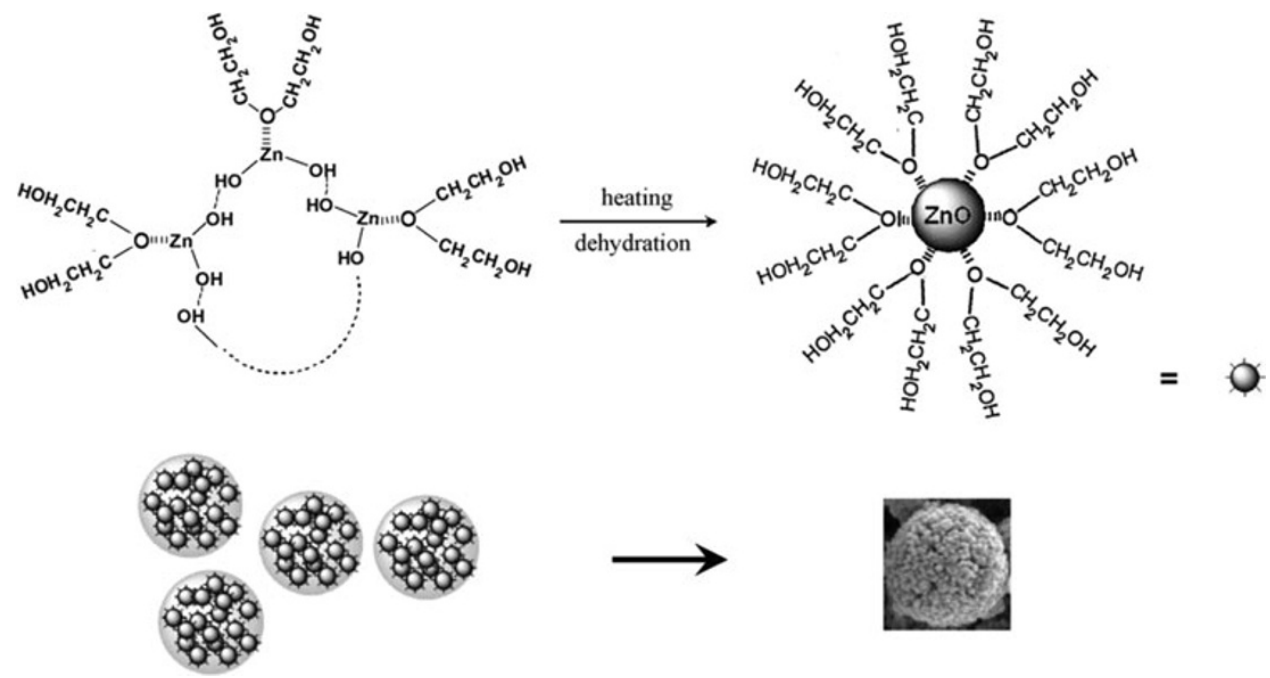

$n \mathrm{Zn}^{2+}+2 n \mathrm{H}_{2} \mathrm{O} \stackrel{\Delta}{\longrightarrow}\left[\mathrm{Zn}(\mathrm{OH})_{2}\right]_{n}+2 n \mathrm{H}^{+}$

$\left[\mathrm{Zn}(\mathrm{OH})_{2}\right]_{n}+n D E G \stackrel{\Delta}{\longrightarrow}\left[\mathrm{Zn}(\mathrm{OH})_{2} \mathrm{DEG}\right]_{n}$

$\left[\mathrm{Zn}(\mathrm{OH})_{2} \mathrm{DEG}\right]_{n} \stackrel{\Delta}{\longrightarrow}[\mathrm{ZnO}-\mathrm{DEG}]_{n}+n \mathrm{H}_{2} \mathrm{O}$

This mechanism can be used to explain the effects of processing parameters on the shape and size of the $\mathrm{ZnO}$ spheres. In the case of molar ratio of $\mathrm{Zn}^{2+}: \mathrm{H}_{2} \mathrm{O}>4$, for instance, the complex compound $\left[\mathrm{Zn}(\mathrm{OH})_{4}^{2-}\right]$ is formed instead of $\left[\mathrm{Zn}(\mathrm{OH})_{2}\right]_{n}$. Therefore, the morphology of $\mathrm{ZnO}$ particles changed to the rod-like shape as showed in Fig. 2d.

For the case when the reaction temperature is below $170^{\circ} \mathrm{C}$ (reaction time up to $24 \mathrm{~h}$ ), the mixed solution became gel-like. No characteristic peak of $\mathrm{ZnO}$ can be detected from the XRD pattern, indicating that $\mathrm{ZnO}$ is not formed due to the insufficient energy provided. When the reaction temperature is above $250^{\circ} \mathrm{C}$, DEG thermal degradation begins to be significant; and this causes the morphology of products to become non-spherical.

\section{Conclusion}

A simple and economic two-stage solution-based method has been developed to synthesize small $\mathrm{ZnO}$ spheres. The product has good uniformity and the diameter of the spheres is in the sub-micrometer range. The morphology and size of the $\mathrm{ZnO}$ spheres can be controlled by the solution composition, reaction temperature, and time. The uniform and submicron sized $\mathrm{ZnO}$ spheres can be used as the photonic crystals to build optoelectronic devices. The effects of different reaction parameters on the formation of $\mathrm{ZnO}$ spheres are investigated and a possible growth mechanism is proposed. In addition, the room temperature PL measurements of the final products were carried out, showing that the $\mathrm{ZnO}$ submicron spheres exhibited a strong UV emission and a broadband green emission in the visible region.

Acknowledgments This research has been supported by both the University of Auckland New Zealand and Nanyang Technological University (NTU) Singapore. The authors would like to thank the technical staffs in the School of Materials Science and Engineering of NTU for various assistances.

Open Access This article is distributed under the terms of the Creative Commons Attribution License which permits any use, distribution and reproduction in any medium, provided the original author(s) and source are credited.

\section{References}

Alver U, Kılınç T, Bacaksız E, Küçükömeroğlu T, Nezir S, Mutlu İH, Aslan F (2007) Synthesis and characterization of spray pyrolysis Zinc Oxide microrods. Thin Solid Films 515:3448-3451

Deng Z, Chen M, Gu G, Wu L (2008) A facile method to fabricate $\mathrm{ZnO}$ hollow spheres and their photocatalytic property. J Phys Chem B 112:16-22

Gao W, Li Z (2009) Nano-Structures of ZnO. Int J Nanotechnol 6:245-257

Khomenkova L, Fernández P, Piqueras J (2007) ZnO nanostructured microspheres and elongated structures grown by thermal treatment of ZnS powder. Cryst Growth Des 7:836-839

Kowalik IA, Guziewicz E, Kopalko K, Yatsunenko S, WójcikGłodowska A, Godlewski M, Dłużewski P, Łusakowska E, Paszkowicz W (2009) Structural and optical properties of lowtemperature $\mathrm{ZnO}$ films grown by atomic layer deposition with diethylzinc and water precursors. J Cryst Growth 311:1096-1101

Ni XM, Zhang YF, Song JM, Zheng HG (2007) Solvent mediated assembly of nickel crystallites: from chains to isolated spheres. J Cryst Growth 299:365-368

Park G, Shindo D, Waseda Y, Sugimoto T (1996) Internal structure analysis of monodispersed pseudocubic hematite particles by electron microscopy. J Colloid Interface Sci 177:198-207

Peng Y, Xu AW, Deng B, Antonietti M, Colfen HJ (2006) Polymercontrolled crystallization of zinc oxide hexagonal nanorings and disks. J Phys Chem B 110:2988-2993 
Peng X, Zang H, Wang Z, Xu J, Wang Y (2008) Blue-violet luminescence double peak of In-doped films prepared by radio frequency sputtering. J Lumin 128:328-332

Ramgir NS, Late DJ, Bhise AB, More MA, Mulla IS, Joag DS, Vijayamohanan K (2006) ZnO multipods, submicron wires, and spherical structures and their unique field emission behavior. J Phys Chem B 110:18236-18242

Seelig EW, Tang B, Yamilov A, Cao H, Chang RPH (2003) Selfassembled 3D photonic crystals from $\mathrm{ZnO}$ colloidal spheres. Mater Chem Phys 80:257-263

Sugimoto T, Wang YS, Itoh H, Muramatsu A (1998) Systematic control of size, shape and internal structure of monodisperse $\alpha$ Fe2O3 particles. Colloids Surf A 134:265-279

Wang ZL (2004) Zinc oxide nanostructures: growth, properties and applications. J Phys Condens Matter 16:R829-R858
Wang X, Hu P, Yuan FL, Yu LJ (2007) Preparation and characterization of $\mathrm{ZnO}$ hollow spheres and $\mathrm{ZnO}$ - Carbon composite materials using colloidal carbon spheres as templates. J Phys Chem C 111:6706-6712

Wu C, Qiao X, Luo L, Li H (2008) Synthesis of ZnO flowers and their photoluminescence properties. Mater Res Bull 43:1883-1891

Xia HB, Yi JB, Foo PS, Liu BH (2007) Facile fabrication of watersoluble magnetic nanoparticles and their spherical aggregates. Chem Mater 19:4087-4091

Yan XD, Li ZW, Chen R, Gao W (2008) Template growth of ZnO nanorods and microrods with controllable densities. Cryst Growth Des 8:2406

Yu H, Zhang Z, Han M, Hao X, Zhu F (2005) A general lowtemperature route for large-scale fabrication of highly oriented ZnO nanorod/nanotube arrays. J Am Chem Soc 127:2378-2379 